\title{
evolución patrimonial y rentabilidad de un grupo empresarial rural: los pereda
}

$(1888-1945)^{\star}$

\section{patrimonial evolution and profitability of an agricultural enterprise: the case of pereda}

(1888-1945)

\author{
Eduardo Martín Cuesta ${ }^{\star} \star$ \\ CONICET, Instituto Interdisciplinario de Economía Política de Buenos Aires, Universidad de \\ Buenos Aires, Buenos Aires, Argentina
}

\section{RESUMEN}

Aquí se presenta un estudio de caso de un gran estanciero argentino: Celedonio Pereda. Su fortuna de Pereda estaba entre las diez más grandes de Argentina hacia 1930. El objetivo de este trabajo es realizar un análisis de la evolución de los negocios y estrategias empresariales de Pereda, entre 1888 y 1945. Esto implica observar en su contexto los patrones de inversión, las finanzas, y las decisiones estratégicas. La evolución del patrimonio y la rentabilidad permite entender las lógicas de acumulación y la vinculación entre la economía argentina y este tipo de empresas en el largo plazo. Para ello se trabaja con las memorias inéditas de Celedonio Pereda (hasta 1928) y con los informes, memorias y balances de la empresa Pereda Sociedad Limitada (entre 1928 y
Abstract

This paper presents a case study of a great Argentine estanciero: Celedonio Pereda and the Pereda Limited. In the late Argentine agro exporter period, Pereda's fortune was among the ten largest in Argentina. The family business became Commercial Society in 1928. The objective of this work is to analyze the evolution of business and corporate strategies of Pereda enterprises, between 1888 and 1945. This involves observing in context patterns of investment, finance, and strategic decisions. The evolution of equity and profitability over this extended period, can understand the logic of accumulation and the link between the Argentina economy and these businesses in the long run. The sources are the unpublished memoirs of Celedonio Pereda (until 1928) and reports and balance

* Agradezco los comentarios y sugerencias recibidas para este trabajo de Carlos Newland, Juan Luis Martirén, Guillermo Banzato, Lionel Barbagallo, y en particular de los dos evaluadores anónimos de la revista.

Fecha de recepción: 22 de diciembre de 2017; fecha de aceptación: 19 de enero de 2019.

$\star \star$ Profesor adjunto interino en la Facultad de Filosofia y Letras de la Universidad de Buenos Aires. Investigador de Consejo Nacional de Investigaciones Científicas y Tecnológicas de Argentina (CONICET). Miembro del Centro de Estudios Económicos de la Empresa y el Desarrollo (CEEED) y del Instituto Interdisciplinario de Economía Política de Buenos Aires (IIEP) de la Facultad de Ciencias Económicas de la Universidad de Buenos Aires.E-mail: cuesta2011@gmail.com 
1945), en conjunto con la evolución de las condiciones macroeconómicas de la Argentina. Estas fuentes permiten un análisis contable, patrimonial, económico y financiero. Así se brinda un nuevo estudio de caso, de fines del siglo XIX a mediados del XX.

Palabras-clave: Historia económica argentina. Estancieros. Historia de empresas. Celedonio Pereda. sheets of the company Pereda S. L. (19281945), together with the evolution of markets and businesses, as well as macroeconomic conditions in Argentina. These sources allow an accounting, patrimonial, economic and financial analysis. This is intended to provide a new case study on the economic agents, in two important periods of Argentina's economic history: the period of agricultural exports and the crisis of the 1930s.

Keywords: Argentine economic history. Argentine estancias. Business history. Celedonio Pereda.

\section{Introducción}

La historiografia argentina ha tenido como uno de los ejes de discusión más controvertidos la figura del agente económico denominado "estanciero". La historiografía discute sobre si su origen está en el período colonial o en el siglo XIX. Sin embargo, no hay lugar a dudas de que fue uno de los protagonistas del período de gran crecimiento y cambio estructural de la economía argentina, de fines del siglo XIX a inicios del XX.

En las últimas décadas del siglo XX la obra de Jorge Sabato (1988) propuso un modelo acerca de los motivos de la gran transformación económica entre fines del siglo XIX y principios del XX, que asentaba su formulación en las características de lo que denominó "clase dominante" 1 . Obviamente, la propuesta de Sabato fue ampliamente discutida por la historiografia, e incluso algunos propusieron denominarlo "modelo sabatino" 2 . El debate que originó la obra de Sabato fue fructífero al poner en discusión las características del proceso de crecimiento económico, los actores, y sus comportamientos. Para Sabato, esta "clase dominante" poseía un comportamiento especulativo, buscando las in-

1 La propuesta de Jorge Sabato se nutrió de un importante acervo previo. Un análisis interesante y profundo al respecto en Pampin (2012).

2 Parte del debate en Eduardo Sartelli (1996), Juan Manuel Palacio (1996) y Fernando Rocchi (1996). Para una perspectiva que incluye a los industriales, ver Jorge Schvarzer (2001). 
versiones más rentables en el corto plazo, evitando el riesgo (estrategia de minimización de riesgo) y las inversiones de largo plazo. Por ello, habría tenido propensión a mantener altos niveles de liquidez. Cabe menciones que Alfredo Pucciarelli (1986) propuso otro esquema interpretativo, en el marco de lo que denominó capitalismo dependiente ${ }^{3}$. En particular, dedica atención a la "gran estancia" (y al agente económico, el estanciero) de la zona pampeana. Entiende que una serie de procesos histórico-económico-sociales derivaron en este tipo de unidad económica, con consecuencias en la estructura. La burguesía terrateniente se habría apropiado del control de la tierra productiva, con alta rentabilidad (en particular de renta diferencial) en un mercado mundial en expansión, con demanda creciente de nuevos productos agropecuarios y altos precios. La gran estancia fue, durante un período concreto, el núcleo dinámico de la modernización y capitalización de la ganadería extensiva ${ }^{4}$.

Si bien el debate aún está abierto, en los últimos años han aparecido estudios de caso de empresarios del periodo de entre siglos que aportan nueva información desde la casuística y confrontan con el modelo sabatino, comprobando algunos de sus puntos y refutando otros. Entre los estudios de caso que se puede mencionar, están los de Ramón Santamarina (Reguera, 2006), Ernesto Tornquist (Gilbert, 2002), Jean Estrogamou (De Nevares, 2012), Eduardo Casey (Newland, 2013), Felipe Senillosa (Hora, 2002, 2003, 2005) y la familia Anchorena (Hora, 2012), entre otros ${ }^{5}$. También los estudios sobre figuras de carácter más fuertemente industrial hacen observaciones al planteo de Jorge Sabato. Por ejemplo, la construcción del Grupo Devoto (Barbero, 2000), el Grupo Tornquist (Gilbert, 2002) o el Grupo Bemberg (Russo, 2009). Desde el

3 La interpretación de Pucciarelli abarca toda la economía argentina, bajo un esquema de capitalismo dependiente, combinado y desigual.

4 En el esquema de Pucciarelli (1986), la acumulación de capital por parte de la burguesía terrateniente fue posible en forma de acumulación de tierras. Lo cual implicaba fuertes y poderosos nexos con la política y la sociedad. En consecuencia, la apropiación privada de la tierra pública, la intensidad y rapidez en la valorización de los activos agropecuarios, y el aumento del precio de la tierra y la renta, derivaron en tendencia hacia el acaparamiento y la especulación.Además, la apropiación de la renta diferencial era posible mediante la modernización de los planteles ganaderos y la tecnología.

5 También se debe mencionar el trabajo de Eduardo Míguez (1985), de gran importancia para entender la propiedad extranjera de la tierra. 
punto de vista del accionar de los terratenientes como grupo u actor económico, se deben mencionar los trabajos de Garavaglia (1999) o Djenderedjian y Schmit (2008), o Schmit (2015), donde se observan los patrones de inversión y estrategias de gestión. Con respecto a los estancieros ganaderos en particular, el trabajo de Sesto (2005) señala el carácter innovador y empresario, de estilo "schumpeteriano". Esto es, visión empresaria, inversión en sectores clave con alto riesgo, innovación, con resultado en altas tasas de ganancia.

Estos aportes historiográficos recientes, enfocados en parte desde la historia de empresas y empresarios, son de gran importancia para conocer las características de los actores de este periodo central en la historia económica argentina, así como también, para continuar disolviendo algunas imágenes algo anquilosadas basadas en miradas impresionistas.

Teniendo en cuenta lo anterior, en este trabajo se hará una aproximación al desempeño económico y financiero del estanciero Celedonio Pereda (1860-1941) . Con el objetivo de observar la evolución del patrimonio y sus patrones de inversión, se trabajará utilizando como fuente principal sus Memorias (Pereda, 1928). En ellas Pereda expresa su deseo de comunicar y dejar un mensaje a sus descendientes, así como demostrar el nivel patrimonial con el cual comenzó, y la riqueza acumulada al momento de dictar sus memorias.

Trabajar con memorias siempre implica tener en cuenta ciertas características intrínsecas de este tipo de fuentes (Durán, 2002). Para el historiador, las memorias, diarios, o autobiografias son un documento excepcional. En primer lugar, son una fuente primaria de "testigos directos". En segundo, el escritor tiene un compromiso con lo que escribe, donde debe expresar "su verdad". Esto no implica ignorar que entre lo vivido y lo elaborado funciona un proceso de reconstrucción, donde el autor interviene de manera decisiva. En gran parte, quien cuenta su vida no es completamente ni objetivo ni desinteresado; es una justificación personal (Gusdorf, 1991). Es una construcción - reconstrucción de su propia identidad. Toda memoria está mediatizada. En primer lugar, por el paso del tiempo. El devenir histórico modifica, tal como menciona

6 La fortuna de Pereda hacia 1930 era una de las diez mayores de Argentina. Una estimación de la misma a valores de 2017 sería de 3 mil millones de dólares norteamericanos. 
el propio Pereda, las opiniones acerca de los sucesos vividos. Se aprecian desde otras perspectivas, diferentes a las de la contemporaneidad. Es una reflexión sobre lo vivido y lo actuado. El segundo elemento es la subjetividad de la perspectiva del actor, ya desde el momento en que transcurren los hechos. La memoria no sólo muestra el punto de vista de Pereda; también el de su generación, que se expresa a través de él. Esto no invalida, sino por el contrario, otorga más valor al ejercicio de la memoria. Ofrece una doble visión al investigador: la del actor, y la del actor en su reflexión. Ayudan a observar el proceso histórico desde la perspectiva de uno de los principales actores, con el agregado de su reflexión casi al final del proceso. Así, estas memorias son el fruto de la reflexión sobre los sucesos, a partir de lo ocurrido, más la experiencia vivida, entre ese tiempo y el momento de la escritura ${ }^{7}$.

También se trabajan las memorias en el contexto económico en que desarrolló sus negocios, y se las interpreta en el marco de la historiografia económica, con lo cual se podrá observar las características empresariales del protagonista. Los datos de los balances, transcriptos por el mismo Pereda, son importantes dada la escasez de registros de estas características, tanto a nivel contable como de gestión. Al mismo tiempo ofrece la visión directa de un protagonista, y permitirá observar sus patrones de inversión y perspectivas acerca de los tiempos que vivió, así como de los resultados que colaboró a construir ${ }^{8}$.

Por otro lado, el trabajo con las Memorias (1888-1928) se complementa con el análisis de los balances de la empresa Pereda S. L. (19281945), cuya disolución se llevó adelante a mediados de la década de 1940. Los estudios sobre beneficios y rentabilidad no son abundantes para este período ${ }^{9}$. Ello no ocurre únicamente en la historiografía ar-

7 Celedonio Pereda escribió una nota dedicatoria a su hijo al comienzo de las $\mathrm{Me}$ morias, fechada en diciembre de 1928. Cierra la dedicatoria con la frase "para que lo poco que yo se respecto de la familia no se pierda, ha sido la idea que he tenido al trazar estos renglones" (Pereda, 1928, p. 1).

8 Las estrategias de inversión de Celedonio Pereda fueron analizadas por Cuesta (2013), para el período $1888-1928$.

9 Principalmente tres autores, Cuesta, Pineda y Lanciotti, han producido estudios que incorporan un análisis de rentabilidad y su relación con el patrimonio o capital: Cuesta (2013, 2014), Lanciotti (2011), Lanciotti y Bartolomé (2014), Pineda (2007). Estudios que incluyen un análisis de rentabilidad son lo de Guy (1988) y Gutiérrez y Korol (1988). También adoptan esta perspectiva Carlos Newland (2015) 
gentina: un grupo europeo de investigación se formó para cubrir la misma vacancia en cuanto a la historia empresarial del viejo continente, con el postulado de que la rentabilidad y desempeño deberían estar en el centro de la disciplina ${ }^{10}$.

A partir de las fuentes y la historiografia presentada, este trabajo pretende aportar un estudio de caso de un empresario ganadero exitoso, y comparar el desempeño de la empresa con otras, tanto agro ganaderas como industriales. Además de describir el desempeño económico de la empresa en relación con el contexto macro y microeconómico, también se analizarán las perspectivas del protagonista, y trabajará con la hipótesis de que la empresa de Celedonio Pereda, que podría pensarse como un caso típico de estanciero rentista, no lo fue tanto, en razón de que Pereda siempre se interpretó a sí mismo como estanciero, que asumió los riesgos de la actividad, invirtió en la misma y mantuvo bajo niveles de liquidez.

Con estos objetivos el trabajo en primer lugar hará una semblanza de la estructura de las fuentes y se esbozará la genealogía familiar y empresarial de los Pereda. En el siguiente apartado se analizarán las medidas que toma Pereda en los convulsionados años que van desde la década de 1880 hasta la Primera Guerra Mundial, que a grandes rasgos podrían interpretarse como la etapa de crecimiento y consolidación de los negocios. El tercer apartado, describirá y analizará el desempeño de la empresa entre 1914 y 1929, momento en que la fortuna alcanza su cenit y podría calificarse como el momento de "madurez" de la misma. En el cuarto, se abordará la rentabilidad y evolución patrimonial de la empresa hasta 1945, comparando con otras empresas. Para finalizar, se presentarán unas breves conclusiones.

\section{Una historia empresaria familiar}

Para explicar el origen de la fortuna de Celedonio Tomás Pereda

en su trabajo "Sobre las estrategias, utilidades y valuación del Grupo Garovaglio y Zorraquín durante el siglo XX" y Newland y Cuesta (2016). Para un trabajo sobre el mercado de capitales, ver Rougier (2012).

10 Disponible en <http://www.lse.ac.uk/economicHistory/BHU/research/performanceEB.aspx $>$. Ver también sobre la escasez de estudios de rentabilidad en Cassis y Brautaset (2003). 
Cuadro 1 - Árbol genealógico de Celedonio Tomás Pereda

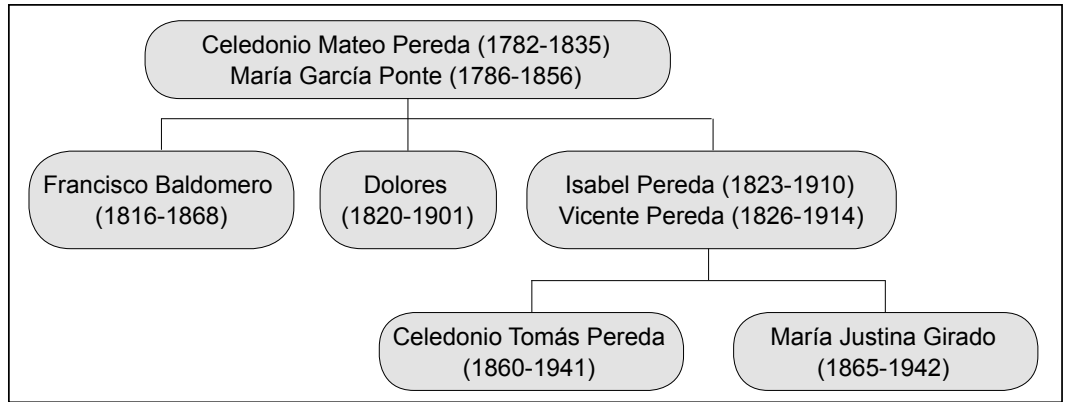

Fuente: según Memorias de Celedonio Tomás Pereda, en Cuesta (2013)^.

* El texto de Cuesta (2013) aborda la historia familiar en detalle, dejando de lado el análisis de patrimonio y utilidades, alcanzando sólo hasta 1928.

(1860-1941) se debe comenzar por el arribo de su ancestro a la Argentina. Celedonio Mateo Pereda (1782-1835) llegó al Río de la Plata, proveniente de España, en la primera década del siglo XIX ${ }^{11}$. Se dedicó al comercio de productos de ultramar, primero como dependiente, $y$ luego como propietario. En 1815 se casó con Mercedes García Ponte. Hasta su muerte, en 1835, desarrolló la actividad comercial en la ciudad de Buenos Aires, al mismo tiempo que abrió una casa comercial en la ciudad de Chascomús, poblado rural a 200 kilómetros de Buenos Aires. Este emprendimiento, como parte de los anteriores, los realizó en sociedad con gestores. Según las costumbres de la época, esto era "habilitar" a un socio no capitalista. La actividad de las casas comerciales en los pueblos rurales era variada, tal como explica Andrea Lluch (2003).

Al momento de su muerte, Celedonio Mateo dejó a su hijo mayor, Francisco Baldomero Pereda (1816-1868) una pequeña fortuna, consistente en participación en casas comerciales urbanas y rurales. La viuda (María García Ponte) retuvo hasta su muerte la propiedad y administración de una decena de fincas urbanas, fuente de rentas seguras. Esto era una costumbre de las familias porteñas: las mujeres quedaban a cargo de los alquileres de los inmuebles urbanos, como forma de asegurarse su independencia mediante un ingreso regular. El patrimonio

11 Según las Memorias (Pereda, 1928) se menciona que habría arribado entre 1805 y 1807. Si bien no se cuenta con el registro preciso de su llegada, aparece su casa y nombre en los padrones de la ciudad desde 1810. 
no se disgregó, ya que la hermana mayor de Francisco, Dolores, no se casó, y su hermana, Isabel, aún era menor y soltera.

Francisco Baldomero amplió la esfera de los negocios heredados. Abrió nuevas casas de comercio: en el pueblo de Dolores (más al sur que Chascomús) y en la ciudad de Gualeguaychú (Provincia de Entre Ríos). En la ciudad de Buenos Aires, con su madre y sus hermanas, compraron más propiedades para renta. También invirtió comprando acciones del Banco Argentino y de la Compañía Primitiva de Gas. De la segunda, fue parte del directorio. Con su prematura muerte, en 1868, dejó una pequeña fortuna, consistente en acciones, propiedades urbanas, y casas de comercio en Buenos Aires, Chascomús, Dolores y Gualeguaychú. También tenía una pequeña cartera de deudores.

Los negocios de la familia pasaron a manos de su primo, Vicente Pereda (1826-1914). Este se había casado con Isabel Pereda (1823-1910), y tenían un hijo: Celedonio Tomás (1860-1941). Vicente tomó a su cargo los negocios, y comenzó la expansión abriendo una casa comercial en el pueblo de Azul, en ese momento frontera sur de la Provincia de Buenos Aires. Continuó la operatoria comercial y financiera del patrimonio familiar, participando en prácticas comerciales con otros españoles, de diversas maneras ${ }^{12}$.

El primer giro en las estrategias de inversión se produce cuando, a partir de su conocimiento del estado de los negocios en el comercio de Azul, comienza a adquirir tierras en la zona. Entre 1869 y 1870 compra dos estancias: "Manantiales" e "Isabel" (la segunda nombrada así por su esposa). Durante la década de 1870 Vicente llevó adelante un proceso de inversiones en ambas estancias, buscando aumentar su rentabilidad. El mayor traspié de la década fueron los conflictos políticos. En primer lugar, la rebelión de Ricardo López Jordán en Entre Ríos (1870-1874), que obligó al cierre de la casa comercial de Gualeguaychú. En segundo lugar, la rebelión de Bartolomé Mitre (1874), que impactó en los negocios en la Provincia de Buenos Aires, y causó la quiebra del Banco Argentino, del que poseían acciones, además de ser cliente. Justamente, ante la quiebra del Banco,Vicente tomó como parte del pago una estancia en la Provincia de Buenos Aires: "La Colorada” en 1875.

12 Estas prácticas comerciales son muy bien descriptas, para otros casos, por Lluch (2009). 
La estructura de los negocios familiares ya estaba delineada para fines de la década de 1870: inversiones en propiedad urbanas, casas comerciales en la ciudad de Buenos Aires, Azul, Dolores y Chascomús, y estancias en Azul ${ }^{13}$. También estaba dando comienzo el traspasamiento de la gestión (proceso de largo plazo) de Vicente a su hijo. En 1880, según sus memorias, Celedonio ya estaba a cargo de los negocios familiares. A partir de las fuentes, se descubre que este último había estudiado la carrera de medicina, a la par que mostraba interés en la administración de las propiedades de la familia.

\section{Patrimonio y rentabilidad de la empresa Pereda (1880-1945)}

\subsection{Primera expansión (1880-1914)}

La década de 1880 significó un fuerte impulso para la fortuna familiar, al compás de crecimiento económico de la Argentina. La campaña militar al sur de 1879 había incorporado al territorio nacional una importante cantidad de tierras. Los Pereda participaron de esta expansión de la frontera adquiriendo y poniendo en producción tierras, tanto en las nuevas zonas como en las antiguas. De hecho, participaron plenamente de la llamada "expansión ganadera" de la Provincia de Buenos Aires. La seguridad jurídica y militar, así como la estabilidad política favorecían la inversión (Barsky; Djenderedjian, 2003). Celedonio Pereda incorporó tierras feraces a la producción, patrón característico del crecimiento de la década de 1880 (Cortés Conde, 1979).

A cargo de la gestión, compartida con su padre desde 1880, en 1888 adoptó las nuevas técnicas contables disponibles en la época. Realizó estudios universitarios en medicina y recibió su título de doctor en 1885. Su tesis revela un interés, quizá de moda en la época, sobre la salud pública $^{14}$. Desde la medicina, su interés luego pasaría al problema de las enfermedades de los vacunos, el tratamiento y curación.

13 Es llamativo que luego del cierre de la casa comercial de Gualeguaychú (Entre Ríos), todos los negocios se concentraron en la ciudad y Provincia de Buenos Aires.

14 El tema de la tesis fue la cremación de cadáveres humanos. 
En este sentido, reconoce que sus estudios sobre contabilidad fueron de tanta utilidad como los de medicina (Pereda, 1928). Los primeros le permitieron tener una visión gerencial de la empresa. Los segundos, participar de la llamada "vanguardia ganadera" que realizó el cambio genético de los planteles vacunos pampeanos (Sesto, 2005).

Durante la etapa de expansión, Celedonio Pereda invirtió en el mejoramiento y refinamiento de los planteles vacunos y ovinos. Asimismo, trabajó en diferentes tratamientos para enfermedades, como la "tristeza bovina" ${ }^{15}$. En simultaneo, llevó adelante distintos proyectos en sus tierras para fomentar y expandir la agricultura, tanto de forrajes como de trigo y maíz. Esto implicó grandes inversiones en maquinarias, alambrados, tanques, etc.

Su casamiento con María Justina Girado (el 7 de septiembre de 1886) fue importante a nivel social, ya que con esta unión la familia Pereda se ligó a una familia de raíz más antigua, reconocida en los círculos porteños, además de importante terrateniente ${ }^{16}$. También en este período ingresó a la Sociedad Rural Argentina (en 1884), de la cual formó parte de su comisión directiva varias veces ${ }^{17}$, así como fue tesorero en varias oportunidades ${ }^{18}$. En un nuevo rol, a los negocios se añadió una importante labor filantrópica.

Bajo la conducción de Celedonio, las inversiones comerciales fueron de a poco perdiendo importancia relativa, frente a las inversiones en tierras, tanto por patrimonio como por rentabilidad, en particular hasta la crisis de 1890. Luego tomarían nuevamente importancia los negocios urbanos, por su valorización desde 1905.

15 La "tristeza bovina" es un complejo de dos enfermedades: la babesiosis y anaplasmosis bovina. Los animales infectados pierden peso, presentan daños en el cuero y dificultades en el procreo.

16 Para un análisis del rol de las redes familiares en las elites, ver Balmori,Voss y Wortman (1990).

17 Entre 1887 y 1889, de 1893 a 1897 , de 1901 a 1905 y desde 1916 a 1922.

18 Fue tesorero por primera vez en 1897. 
Tabla 1 - Composición del patrimonio Pereda en relación con el total del activo, 18891914 (en \%)

\begin{tabular}{l|r|r|r|r|r|r}
\hline \multicolumn{1}{c}{} & \multicolumn{1}{c}{1889} & \multicolumn{1}{c}{1894} & \multicolumn{1}{c}{1899} & \multicolumn{1}{c}{1904} & 1909 \\
\hline Inmuebles urbanos & 7 & 9 & 9 & 7 & 16 & 16 \\
\hline Campos & 45 & 40 & 60 & 62 & 47 & 42 \\
\hline Acciones & 11 & 10 & 5 & 2 & 3 & 4 \\
\hline Ganado & 32 & 24 & 19 & 23 & 26 & 34 \\
\hline Deudores & 4 & 14 & 4 & 5 & 6 & 2 \\
\hline Efectivo y bancos & 1 & 2 & 3 & 1 & 2 & 1 \\
\hline & & & & & & \\
\hline Pasivo & 2 & 8 & 2 & 6 & 3 & 7 \\
\hline
\end{tabular}

Fuente: elaboración propia a partir de Cuesta (2013).

En 1878 Celedonio compró tierras en la zona de Lincoln, mediante remate; allí se crearon las estancias "13 de Abril" ${ }^{19}$ y "El Infierno". Al año siguiente se compraron tierras en Trenque Lauquen, al oeste de la Provincia de Buenos Aires, recién incorporadas efectivamente al territorio productivo. Allí se fundaron las estancias "San Baldomero" y "Nueva Castilla". En esta compra también participó Vicente como asesor, señalando las ventajas y desventajas de estas tierras. El giro terrateniente que dio Celedonio a los negocios de la familia fue claro. Según relata él mismo: "Dicen que desde muy corta edad manifesté gusto por los negocios de campo, pues cuando me preguntaban que iba a ser siempre decía que estanciero" (Pereda, 1928, p. 179).

En 1884 compró las estancias "La Asunción” y "La Encarnación”. En 1895 compró "Villa María”, en el partido de Ezeiza (Provincia de Buenos Aires). En 1900 compró la estancia "La Unión” en el partido de Gral. Madariaga, y al año siguiente compró las tierras linderas del "13 de Abril".

Más tarde compró estancias en otras provincias argentinas: en 1910 la estancia "El Tunal” en la Provincia de Salta, y en 1911 "La Guampita" en la Provincia de Santa Fe.

En varias de sus estancias, ante el auge de los cereales, realizó emprendimientos de cultivos de trigo y maíz, tanto de manera directa como

19 Llamada así por la fecha de nacimiento de la esposa de Celedonio Tomás. 
arrendando a agricultores. La visión de Celedonio con respecto a los ciclos de los negocios era clara:

no debe descorazonarse ni desalentarse porque se presenten años malos, pues todos los negocios tienen sus alternativas, que son muy frecuentes en la ganadería y en la agricultura, pues cuando uno de estos renglones de la producción que son los más importantes en nuestro país mejora el otro decae. (Pereda, 1928, p. 185)

Con respecto a la cartera de valores mobiliarios, Celedonio incorporó al portfolio acciones de diversas empresas. Frente al impulso a la instalación de gas domiciliario, en 1886 adquirió más acciones de la Compañía primitiva de Gas. En 1887 suscribió acciones del Mercado Central de Frutos, que llegaría a ser el depósito de lanas y cueros más grande del mundo en la década de 1890 (Newland, 2012). En 1896 junto con otros estancieros fundó un frigorífico de capitales nacionales, que luego fue vendido a capitales extranjeros. Con la modernización del transporte urbano de la ciudad de Buenos Aires, compró acciones de "Tranvías eléctricos del sur". En varias de estas empresas formó parte del directorio, participando activamente en la gestión (en particular del Mercado Central de Frutos y las empresas de la ciudad de Azul).

También hizo inversiones de poco rédito. Compró acciones de la Compañía "Chocloína”, la "Sociedad de Fundición de Hierro y Acero", "La Africana" y de "La Higiénica" (entre 1895 y 1910). Las cuatro fueron un fracaso. Otras inversiones mobiliarias fueron más seguras, como las acciones del Banco Popular Argentino y del Banco Español en 1901 y del Banco Comercial de Azul en 1908. Para este momento, ya superada la crisis de 1890, los bancos estaban firmes (Regalsky, 1994). También fundó y compró acciones de la Cooperativa de Seguros "La Azuleña" (de la localidad de Azul).

En paralelo, incrementó la cantidad de propiedades urbanas. Entre estas, en 1917 compró el terreno sito en la calle Arroyo donde construyó más tarde el "Palacio Pereda"20.

20 Entre otras propiedades adquiridas en la ciudad de Buenos Aires figuran las ubicadas en las siguientes direcciones: Salta 673 y Piedras 975 (1878); Belgrano 935 (1896); Tacuarí y Moreno (1897); Tacuarí 92 (1901); Suipacha y Tucumán (1902); Charcas 1.173 (1913). 
La crisis de 1890 parece no haber afectado los negocios de Pereda. Quizá, por el rol destacado que el empresario daba a la liquidez financiera. Según la historiografia, justamente ese fue uno de los principales problemas. La depreciación del papel moneda, en correlato directo a la apreciación del oro, fue la característica de la crisis económica (Cortés Conde, 1989; Gerchunoff; Rocchi; Rossi, 2008). Es posible que la crisis configurara su memoria acerca de las estrategias de inversión:

Hay que cuidarse de los años de prosperidad, pues como en esta época todo marcha en auge se entusiasma y quiere agrandar y aumentar los negocios, lo que hace que los artículos salgan perdiendo valor del poder de adquisición y cuando la reacción llega y se producen los cambios el artículo baja de precios y el dinero se valoriza [...] la conducta seguida por la casa ha sido tener dinero en abundancia para poder afrontar cualquier negocio sin inconvenientes. (Pereda, 1928, p. 186)

Pereda aconseja "manejo cuidadoso de las inversiones y preferencia por la liquidez". La expansión en tierras fue constante, en la Provincia de Buenos Aires hasta 1920, y en otras provincias desde 1900. Las incorporaciones de propiedades urbanas le otorgaban solidez. Las inversiones en acciones fueron secundarias en el patrimonio. El espectacular crecimiento del patrimonio se explica por las altas utilidades (Gráfico 1), que se reinvertían.

La reinversión de las ganancias era muy importante. Celedonio señaló: "si las rentas pasan las necesidades de la vida familiar, acumular estas para aumentar el capital" (Pereda, 1928, p. 185). Ello se observa en los datos en la alta tasa de reinversión de las utilidades, tanto en tierras, inmuebles, como en capital fijo y tecnología productiva (alambrados, edificios, molinos, jagüeles, desagües, etc.) y en veterinaria y genética (corrales, vacunas, etc. $)^{21}$. Los informes de inversión sobre equipos priman a la hora de la evaluación del rendimiento de los negocios ${ }^{22}$ (molinos, bombas, norias, sembradoras, cosechadoras, etc. $)^{23}$.

\footnotetext{
21 La inversión en capital fijo la ha señalado Newland (1999), a nivel macroeconómico.

22 Por ejemplo, para "El Tunal” (Pereda, 1928, p. 143).

23 Como la compra de cosechadoras de maíz para "San Baldomero" (Pereda, 1928, p. 122).
} 


\subsection{Segunda expansión (1914-1929)}

Entre 1912 y 1914, la evolución de las utilidades (Gráfico 1) y la composición del patrimonio (Tablas 1 y 2 y Gráfico 3) muestran un cambio. Si bien continúan las inversiones en acciones, como las del frigorífico "Sansisena" en 1917, comienza a declinar la compra de tierras en la Provincia de Buenos Aires, y se expande la adquisición de tierras en otras provincias ${ }^{24}$.

La fortuna de la familia Pereda queda consolidada en este período, siendo reconocido como uno de los grandes terratenientes de la Argentina, dentro de los cinco mayores de la década de $1920^{25}$. Como miembro importante de la Sociedad Rural Argentina, y empresario ganadero, participó del proceso de refinamiento de las razas vacunas. Su estancia "13 de Abril" fue utilizada para experimentar tratamientos para diversas enfermedades bovinas. En 1922 Pereda presentó el primer proyecto de registro de haciendas puras.

La composición del patrimonio en este período muestra el incremento en las propiedades urbanas, tanto por cantidad como por valorización. Las tierras, que aumentaron su valor en el período previo, se estabilizan en un 40\% del patrimonio. El ganado desciende en proporción, por la estabilización de su precio, así como el aumento en el rubro financiero (deudores).

Los resultados de la empresa, registrados desde 1888 hasta 1928 en los libros de contabilidad de Pereda (y trascripto en las Memorias), y desde 1928 en los balances presentados ante las autoridades, fueron siempre positivos.

La evolución de las utilidades deja observar las tres etapas. La primera entre 1888 y 1912, la segunda entre 1912 y 1920, y la tercera entre 1921 y 1945. En la primera etapa, las utilidades no superaron el millón de pesos, con cierta volatilidad (no muy pronunciada), con tendencia

24 En 1918 se incorporan al patrimonio campos en la Provincia de San Luis, en 1920 la estancia "El Naranjal” en la Provincia de Entre Ríos y la estancia "El Clavo" en la Provincia de San Luis y en 1925 campos madereros en "Abra Grande" (Provincia del Chaco).

25 La familia Pereda es mencionada entre los principales miembros de la elite (de la que formaban parte los Anchorena, Senillosa, Santamarina, o Duhau), como se observa en los trabajos de Roy Hora (2002) o de María Sáenz Quesada (1980). 
Tabla 2 - Composición del patrimonio Pereda en relación con el total del activo, 1914-1927 (en \%)

\begin{tabular}{l|c|c|c|c}
\hline \multicolumn{2}{c}{1914} & 1919 & 1924 & 1927 \\
\hline Inmuebles urbanos & 16 & 24 & 23 & 26 \\
\hline Campos & 42 & 34 & 41 & 41 \\
\hline Acciones & 4 & 3 & 3 & 5 \\
\hline Ganado & 34 & 27 & 19 & 14 \\
\hline Deudores & 2 & 11 & 11 & 11 \\
\hline Efectivo y bancos & 1 & & 2 & 1 \\
\hline & & & & 1 \\
\hline Pasivo & 7 & 5 & & \\
\hline
\end{tabular}

Fuente: elaboración propia a partir de Cuesta (2013).

Gráfico 1 - Utilidades anuales de la empresa Pereda en \$ oro, 1889-1945

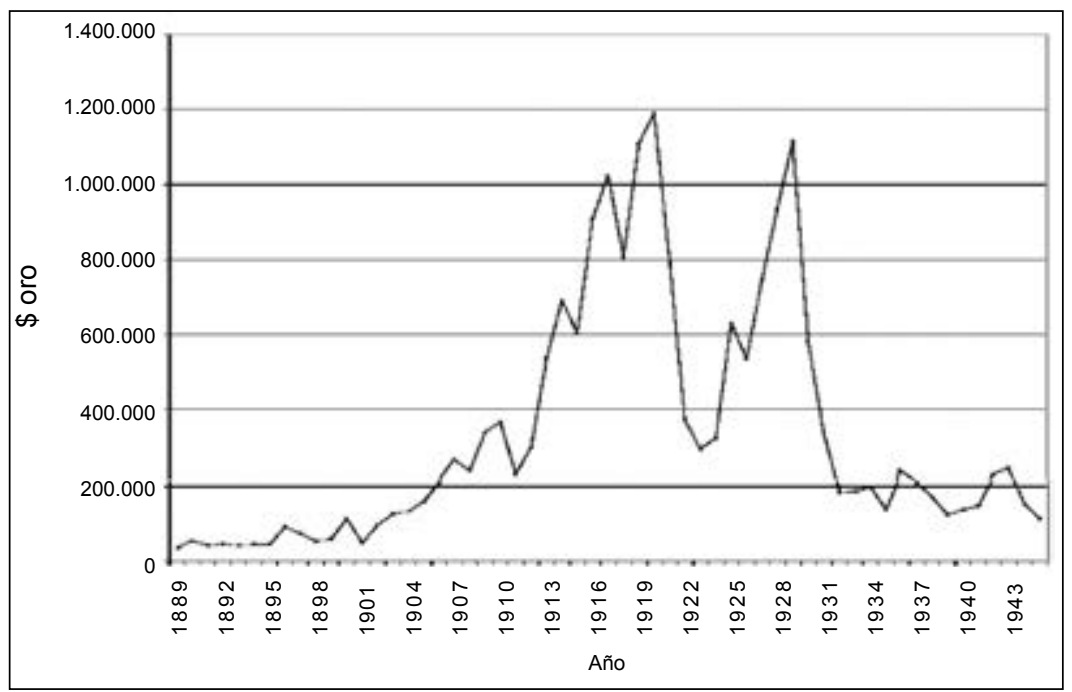

Fuente: elaboración propia a partir de las Memorias de Celedonio Pereda (1928), Cuesta (2013), Boletín de la Bolsa de Comercio de Buenos Aires (1888-1960).

positiva. En la segunda, se observa un rápido y vertiginoso crecimiento hasta 1920 , en que se triplican las utilidades hasta alcanzar un poco menos que los 3 millones de pesos. Pero esta segunda etapa también es volátil, con altas utilidades, pero también con fuertes variaciones. Durante la Primera Guerra Mundial las utilidades crecieron, por el alza en el precio de los productos ganaderos, resultado del crecimiento de la 
demanda europea. La tercera etapa inicia en la década de 1920 con una crisis ganadera, que se refleja en la caída de las utilidades. La crisis de 1920-1922, producto del fin de la Primera Guerra Mundial, la baja de la demanda y en los precios impactó fuertemente en el sector ${ }^{26}$. Sin embargo, durante los siguientes años se recuperó el mercado y hacia fin de la década las utilidades se recuperaron.

La crisis de 1930 impactó en las rentas, produciendo alta volatilidad en los ingresos hasta la Segunda Guerra Mundial, que inició un período de alza hasta 1943. La empresa comenzó su liquidación en 1944, finalizando su operación en 1945, lo que se observa en la caída de la tendencia en el gráfico ${ }^{27}$.

Es interesante observar que la evolución de las utilidades de la empresa Pereda guarda una gran correlación con la evolución de las exportaciones argentinas, que se presentan en el siguiente gráfico.

Gráfico 2 - Índice de exportaciones argentinas en valores, 1889-1945

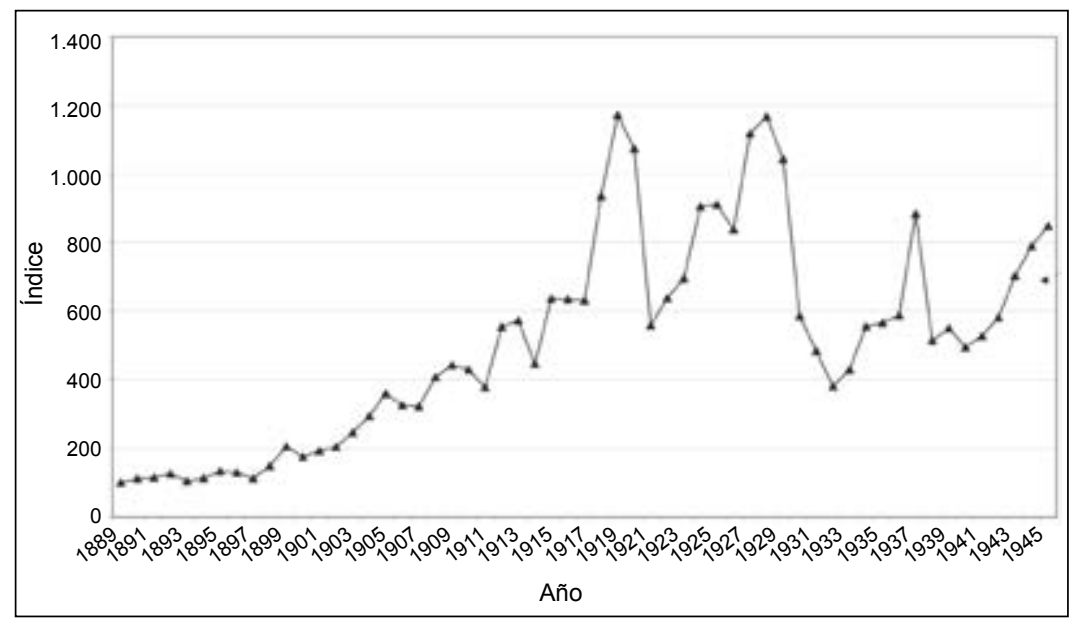

Fuente: elaboración propia a partir de Cortés Conde (2007).

Como se ha presentado en la anterior cita de Pereda, las utilidades fueron incorporadas al capital de la empresa, mediante la compra de

26 Para un análisis de la evolución del sector ganadero argentino durante el período, ver Hanson (1937).

27 Celedonio Mateo falleció en 1941, sobreviviendo su empresa algunos años más. 
diferentes activos, de los cuales los principales fueron las propiedades inmobiliarias (en primer lugar, rurales y luego urbanas), ganado y en tercer lugar activos financieros. El crecimiento del patrimonio, en gran correlación con el crecimiento de las utilidades, se observa en el Gráfico 3. La empresa se financiaba por reinversión de utilidades, no mediante uso del crédito (ver el porcentaje de pasivo en Tablas 1 y 2).

Gráfico 3 - Patrimonio neto de la empresa Pereda en \$ oro, 1889-1945

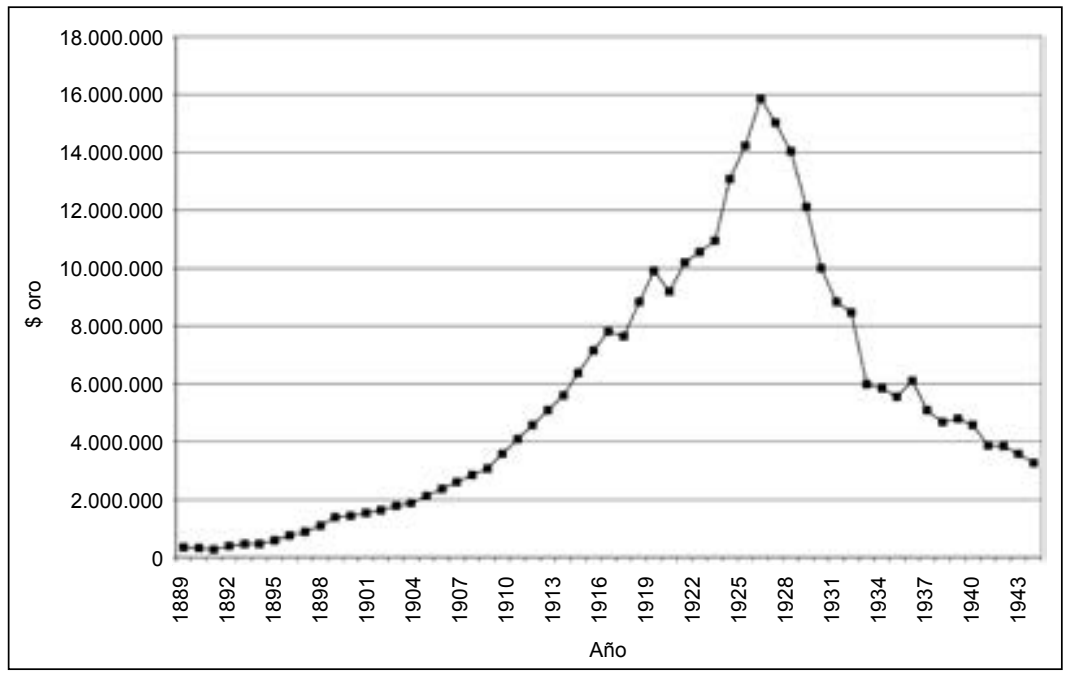

Fuente: elaboración propia a partir de las Memorias de Celedonio Pereda (1928), Cuesta (2013), Boletín de la Bolsa de Comercio de Buenos Aires (1888-1960).

Se explica el crecimiento constante del patrimonio hasta 1927 por la reinversión de las utilidades y la valorización de las propiedades urbanas y rurales, del ganado, así como por el crecimiento del capital fijo ${ }^{28}$ (maquinarias, tanques, alambrados, etc.). Es interesante señalar el crecimiento continuo del patrimonio, sin sufrir la volatilidad del sector (en especial la de la Primera Guerra Mundial y la crisis de 1920-1922). Entre 1927 y 1930 cae el patrimonio neto, como resultado de la transformación en Sociedad Limitada, dado que algunos activos inmuebles urbanos no se traspasan a la misma, y por la caída en el valor de la tierra. De allí en adelante, se observa el impacto de la crisis de 1930, y se es-

28 Se entiende aquí capital fijo en su definición contable. 
tabiliza hasta 1945, año de disolución de la empresa. Como se ha expuesto, el valor de la empresa también estaba vinculado al precio de su principal activo fijo: la tierra.

Gráfico 4 - Índice del precio de la tierra ganadera en la Provincia de Buenos Aires, 19161945 (base $100=1916$ )

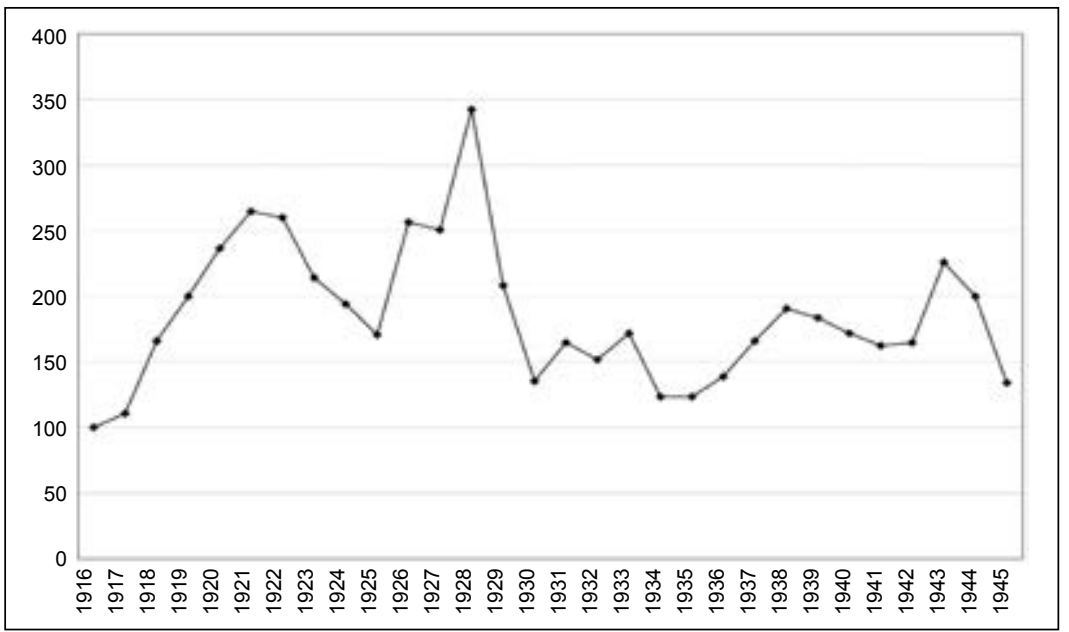

Fuente: elaboración propia a partir de Ras y Lewis (1980).

La evolución del precio de la tierra muestra dos procesos de alza y caída. Uno entre 1916 y 1925, explicado por la Primera Guerra Mundial y luego por la crisis del ganado ${ }^{29}$, y otro 1925 y 1930 explicado por la recuperación ganadera y la caída en la crisis de 1929.

Los precios de la tierra se estabilizaron en la década de 1930, a valores poco muy superiores a los de 1916. Recién con la Segunda Guerra Mundial volverán a subir de precios las tierras bonaerenses. La crisis de 1929 fue un punto de inflexión en los precios.

Justamente en 1929 la empresa se transformó en Sociedad de Responsabilidad Limitada; Celedonio seguramente estimaba que era una manera efectiva de consolidar el patrimonio familiar frente a la posible dispersión entre sus seis herederos.

29 Para un análisis detallado del impacto de la Primera Guerra Mundial en el precio del ganado y la tierra, ver Hanson (1937). 
El éxito de la empresa dependió de muchos factores, además de los precios de la tierra y el ganado. Por ejemplo, uno de los grandes problemas eran las enfermedades del ganado. Pereda no sólo se preocupó por la zoonosis de su ganado, sino que también invirtió en tratamientos e investigaciones para ayudar a todo el sector. Figura como fundador, y financiador, del Instituto Pasteur de Buenos Aires.

\subsection{Madurez y cierre (1929-1945)}

Los últimos 16 años de la empresa son representativos de la madurez de la misma, así como del devenir de la economía argentina. Las utilidades caen a consecuencia de la crisis mundial (Gráfico 1) entre 1929 y 1933, para luego estabilizarse a mediados de la década. Recién con la Segunda Guerra Mundial las utilidades vuelven a ascender, transitoriamente, por las condiciones de la economía mundial. El comportamiento y nivel de las utilidades, explica que ya no se reinviertan, y en consecuencia se deteriore el patrimonio neto (Gráfico 3), acompañando los precios (Gráfico 5).

Gráfico 5 - Índice del precio del novillo en Buenos Aires, 1893-1945 (base $100=1893$ )

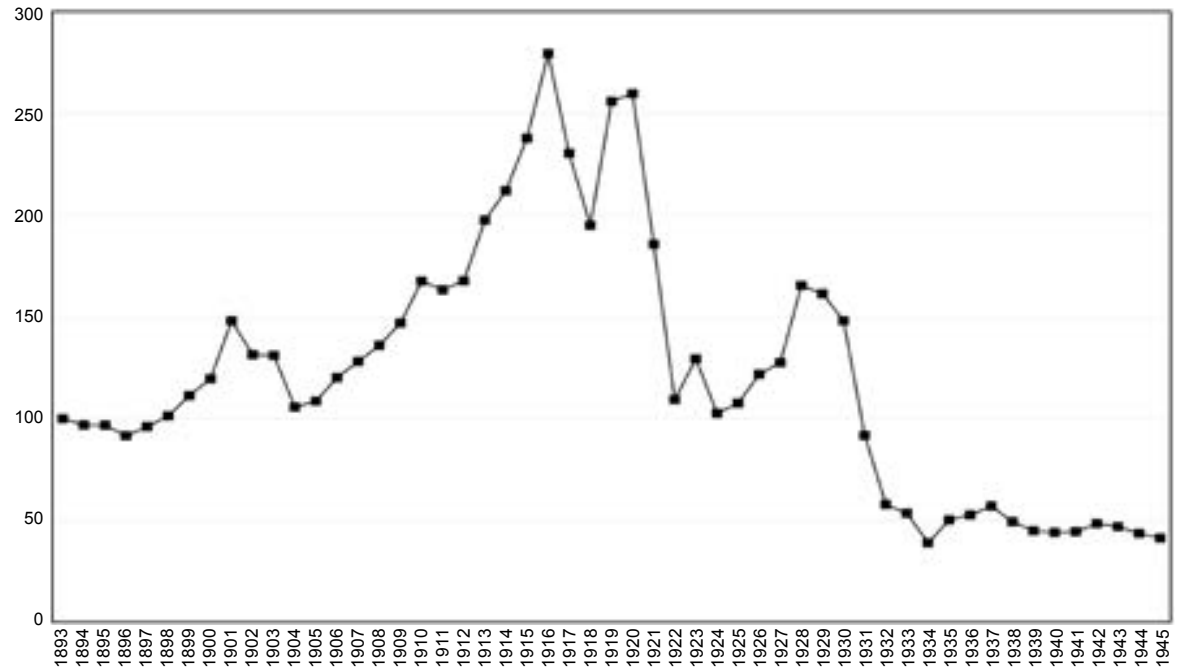

Fuente: elaboración propia a partir de los datos de Álvarez (1928), sobre el precio en \$ oro del novillo de exportación en el mercado de Buenos Aires. 
En parte, el largo ciclo de utilidades hasta 1921 (Gráfico 1) y la posibilidad de reinversión y crecimiento del patrimonio (Gráfico 3), estuvo marcado por el sostenido incremento en el precio de los vacunos (Gráfico 5). Estos triplicaron su precio entre 1893 y 1917, generando amplias utilidades. Luego, con la crisis ganadera de 1920-1922, por la caída de la demanda y el exceso de stock vacuno, los precios cayeron. Recién se recuperarían a fines de la década.

Para completar el análisis, contando con las utilidades y el patrimonio neto se ha calculado la rentabilidad de la empresa sobre el patrimonio, que se presenta en el gráfico a continuación.

Gráfico 6 - Rentabilidad (utilidades/patrimonio neto) de la empresa Pereda (en \%)

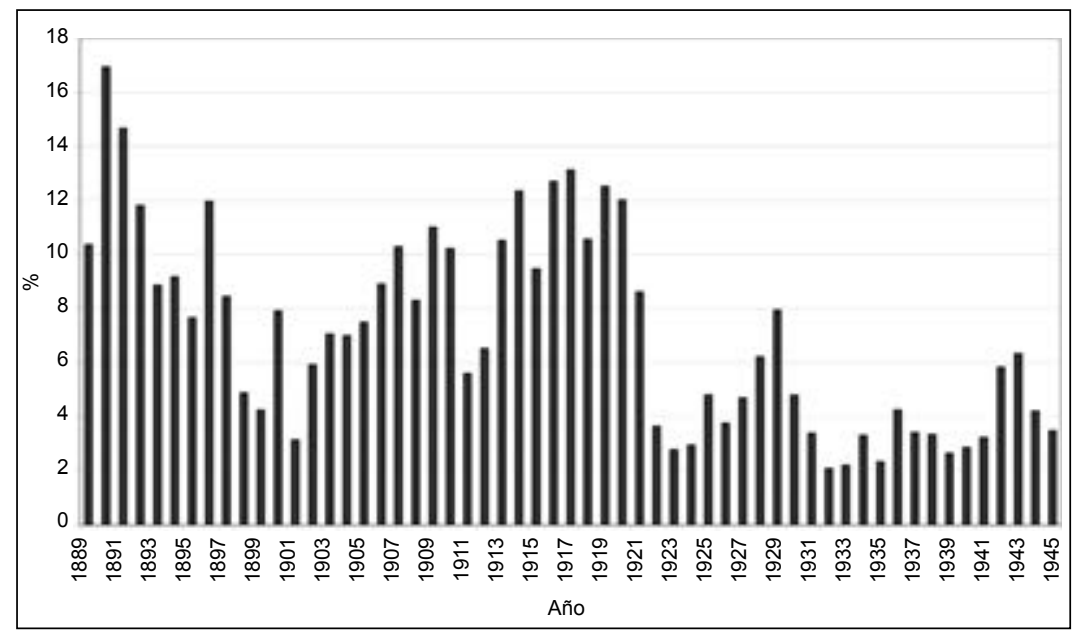

Fuente: elaboración propia a partir de las Memorias de Celedonio Pereda (1928), Cuesta (2013), Boletín de la Bolsa de Comercio de Buenos Aires (1888-1960).

La evolución de la rentabilidad es sugerente de la evolución de la actividad en general. Entre 1889 y 1911 las altas rentabilidades permiten la reinversión permanente en tierras, ganado y materiales, que apalancan mayores ingresos. El incremento en la producción correlaciona con la demanda internacional, y por ende en precios e ingresos. La década de 1910, con la empresa en plena actividad y el contexto bélico de la Primera Guerra Mundial, generó utilidades record (Gráfico 1), aumento del patrimonio, y rentabilidades elevadas y sostenidas. 
Con la crisis ganadera de 1920-1922, cae el precio del ganado, y se acabó el ciclo de alta rentabilidad. Las utilidades se reinvierten, y sigue creciendo el patrimonio, pero con una rentabilidad a niveles bajos. Sólo algunos años excepcionales permite rentabilidades superiores al 6\%.

La década del 1930 muestra un estancamiento del patrimonio neto, así como de la rentabilidad. Sólo la Segunda Guerra Mundial, y su demanda, permiten utilidades por encima del $4 \%$, quedando el promedio en el 3\% anual.

En este indicador también se observan dos períodos bien definidos. El primero entre 1889 y 1929, de altas y muy volátil rentabilidad. El segundo, de menor escala, y más estable. Esto se corresponde con lo observado en el Gráficos 1 y 3, donde el crecimiento del patrimonio es después de la década de 1920. Las altas rentabilidades entre el inicio de la serie y el fin de la Primera Guerra Mundial explican el gran crecimiento del patrimonio neto (Gráfico 3).

A modo de hipótesis, el tiempo de las grandes ganancias (y fortunas) de las explotaciones agropecuarias era pasado; Pereda Sociedad Anónima Limitada no fue la excepción. Trabajos como el de Newland (2015) demuestran que la década de 1920 fue la última de grandes rendimientos del sector agropecuario, y el primero de sector industrial.

Se dispone de trabajos comparativos de otras empresas del período, que muestran las altas rentabilidades de las empresas industriales frente a las agropecuarias, como se muestra en la Tabla 3 .

Tabla 3 - Índice de rentabilidad (utilidades/patrimonio neto) del Mercado Central de Frutos, Bunge y Born y Garovaglio y Zorraquín (en \%)

\begin{tabular}{c|c|c|c}
\hline \multicolumn{2}{c}{ MCF } & ByB & GrZ \\
\hline $1926-1932$ & $7 \%$ & $15 \%$ & $12 \%$ \\
\hline $1933-1941$ & $5 \%$ & $8 \%$ & $5 \%$ \\
\hline $1942-1948$ & $8 \%$ & $15 \%$ & $12 \%$ \\
\hline $1949-1955$ & $6 \%$ & $11 \%$ & $11 \%$ \\
\hline
\end{tabular}

Fuentes: Newland; Cuesta (2016); MCF - Mercado Central de Frutos, empresa vinculada a la agroexportación; GyZ - Garovaglio y Zorraquín, empresa de comercialización de productos agropecuarios; $\mathrm{ByB}$ - Bunge y Born, empresa de comercialización de productos agropecuarios, que en la década de 1920 comienza su diversificación hacia el sector industrial (química, molinos, etc.). 
Según Newland (2017), el cálculo agregado de rentabilidad de los sectores (Gráfico 7) ${ }^{30}$, demuestra la caída de la rentabilidad agropecuaria en la década de 1920, con piso en los primeros años de la gran depresión. Si bien el sector se recupera a fines de los 1930, y un poco durante la Segunda Guerra Mundial, no supera los niveles la década de 1920. En cambio, el sector industrial crece de manera significativa en la década de 1920, y si bien cae con la crisis de 1930, se mantiene siempre por encima de la rentabilidad agropecuaria. Incluso en los años de la Segunda Guerra Mundial, crece más que el sector agropecuario.

Gráfico 7 - Rentabilidad sectorial (agropecuaria e industrial) en Argentina, 1926-1945 $(\text { en } \%)^{\star}$

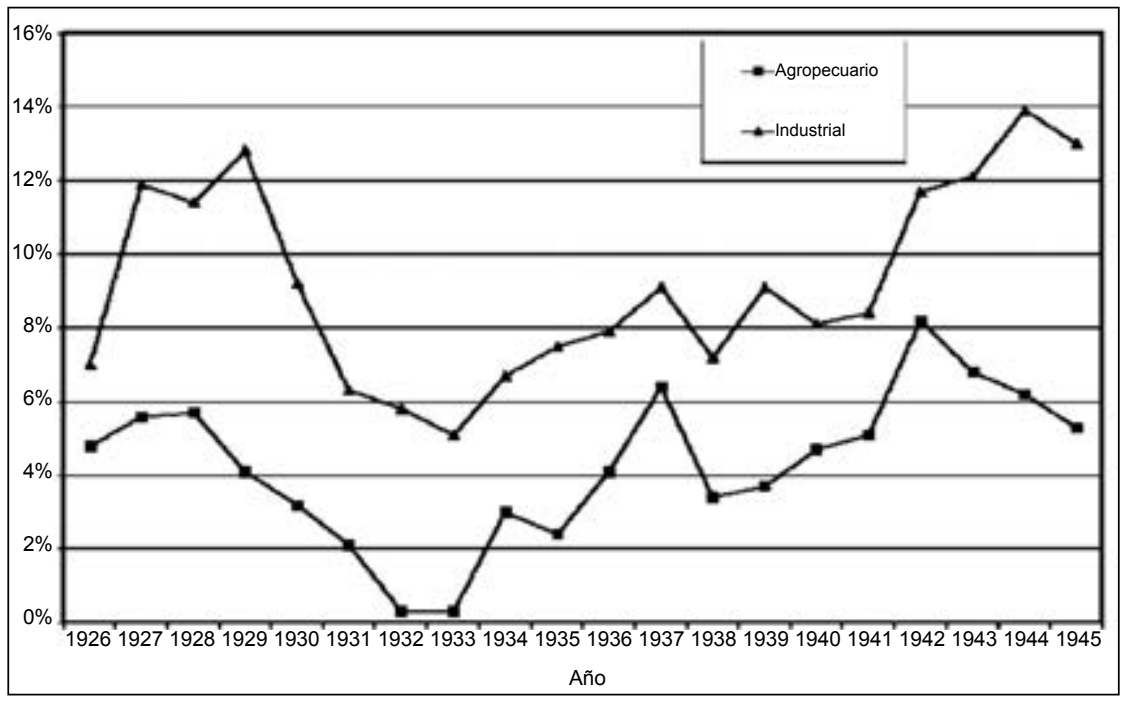

Fuente: elaboración con datos de Newland (2015).

* Se entiende la rentabilidad como la relación entre utilidades y patrimonio neto.

\section{Consideraciones finales}

En este trabajo se ha presentado un análisis del derrotero empresarial de un gran estanciero argentino de fines del siglo XIX y principios del

30 Newland (2015) realiza este cálculo a partir de un minucioso y exhaustivo trabajo sobre diferentes empresas de cada sector, entendiendo este índice como el cociente entre utilidades y patrimonio neto. 
XX: Celedonio Pereda. Esto fue posible gracias a la disponibilidad de fuentes como las memorias y balances de la empresa "Pereda Ltda. Sociedad Anónima" (1928-1945), así como de las Memorias del propio estanciero. El estudio del caso, en su contexto histórico, social y económico, es útil para adentrarse y esclarecer el período, así como para contrastar con las propuestas teóricas acerca de las condiciones de los agentes económicos.

La historiografia sobre estancieros y/o empresarios para el período denominado "Argentina Próspera" (1880-1930) es abundante. Observando el caso a la luz de estas propuestas, no parecería el caso de un estanciero "absentista" o "rentista". Dada su participación en el cambio genético vacuno, se podría encuadrar en la tesis de Carmen Sesto sobre la "vanguardia ganadera".

Por otro lado, la historia empresarial de Pereda, y los resultados económicos financieros, se pueden asociar a los precios y condiciones de la economía argentina, así como a las trayectorias familiares de la elite ya conocidas. Desde el arribo de Celedonio Mateo a Buenos Aires a principios del siglo XIX, hasta Celedonio Tomás en el siglo XX, se observa un "camino" de ascenso social. Comenzando en las actividades mercantiles, urbanas y rurales, a la adquisición de tierras e inmuebles, la explotación ganadera, y finalmente la mansión y el acceso a la elite porteña ${ }^{31}$. El casamiento de Celedonio con María Girado extendió la red familiar, incrementando redes y posibilidades ${ }^{32}$. Miembro de la elite terrateniente, la prosperidad de los Pereda se expresó en la construcción de un lujoso casco en la estancia "Villa María"33 , y del "Palacio Pereda" en la ciudad de Buenos Aires ${ }^{34}$.

31 En cierto modo, es el patrón de inversiones que analiza Susan Socolow (1991) para las fortunas de origen colonial, como los Anchorena. Otros casos similares son las familias Duhau o Estrogamou.

32 La familia Pereda - Girado es entendida como una red familiar de la elite por autores como Balmori,Voss y Wortman (1990).

33 La Estancia "Villa María”, comprada a fines del siglo XIX para invernada, comenzó a tomar otro impulso en el siglo XX. En 1917 Celedonio contrató al paisajista Benito Carrasco (discípulo de Carlos Thays), quien diseñó un parque de 74 hectáreas. Y en 1919, encargó a Alejandro Bustillo la construcción del casco, con estilo Tudor normando. Así "Villa María" se transformó en residencia de verano.

34 Los terrenos fueron comprados en 1917, y la construcción comenzó en 1919. El proyecto fue encargado al arquitecto francés Louis Martin, quien ya había realiza- 
La descripción de los ciclos vitales y familiares en su aspecto empresario dejan ver un patrón muy conocido en la historia económica latinoamericana. En primer lugar, un inmigrante que se inserta en el comercio a pequeña escala, para luego ir incrementando su capital mediante asociaciones y vinculaciones. Desde el comercio, en una segunda etapa se consolida el capital con la inversión en tierras. Como última etapa, se observan las inversiones urbanas y en activos financieros. Este "patrón" de evolución económica y vital ya fue observado para el siglo XVIII y XIX por Socolow (1991), por Balmori, Voss y Wortman (1990) para otros países de la región, y es similar al derrotero de Santamarina (Reguera, 2006).

Las fuentes del caso Pereda ilustran acerca de las características de la gestión y crecimiento patrimonial entre 1888 y 1945. Al igual que en el caso del estanciero Santamarina, a la muerte de Celedonio Pereda la empresa fue liquidada por sus herederos ${ }^{35}$.

El análisis de la evolución del patrimonio permite observar tres períodos. Uno desde fines del siglo XIX hasta inicios del XX, en que la reinversión de las utilidades permitió una capitalización en tierras y ganado, con lo cual se incrementó el patrimonio. Esto implicó ingresar en la apuesta al cambio genético vacuno, con la incertidumbre que ello implicaba ${ }^{36}$. Pereda invirtió en planteles, instalaciones, tecnología veterinaria, etc. Una segunda, entre inicios del siglo XX y 1930, en que el crecimiento del patrimonio continúa, pero la fluctuación en los precios del ganado y el contexto económico hacen que las utilidades sean volátiles, y el patrimonio se incremente a una tasa menor que en el período anterior. La bonanza de los precios vacunos con la Primera Guerra Mundial generó altas utilidades. En este período se continúa con la reinversión, en especial en tecnología ganadera y veterinaria, así como también incorpora tierras fuera de la Provincia de Buenos Aires.

do otros edificios. Celedonio le solicitó que construyera sobre modelos franceses, de edificios que lo habían impactado. El edificio fue finalizado por el arquitecto Julio Dormal, en 1936. Fue comprado por el gobierno de Brasil en 1944, siendo actualmente la Embajada de la República Federativa del Brasil (calle Arroyo 1130, Ciudad Autónoma de Buenos Aires).

36 Según Sesto (2005) la inversión en reproductores y planteles era considerable, e implicaba grandes cuidados y trabajos de adaptación, no siempre exitosos. 
Se observó que el activo de la empresa estuvo conformado en un gran porcentaje por tierras y ganado, el cual fue fluctuando de acuerdo con el mercado. Las inversiones inmobiliarias urbanas muestran una evolución a lo largo del tiempo, de acuerdo con los patrones familiares habituales para la época. Asimismo, se pudo comprobar la existencia de inversiones financieras que, si bien no importaban en gran medida sobre el total del activo, eran significativas en valores nominales. Ello denota que, si bien había inversiones en agricultura, así como en bonos y acciones, la mayor parte del patrimonio y los negocios eran ganaderos; es decir, era una empresa poco diversificada. También se observa que Pereda no tenía una gran reserva de liquidez sobre su capital ${ }^{37}$.

El éxito de la empresa dependió de muchos factores, además de los precios de la tierra y el ganado. Por ejemplo, uno de los grandes problemas eran las enfermedades del ganado. Pereda fue uno de los fundadores, y financiadores, del Instituto Pasteur de Buenos Aires. Otros temas que preocupaban a la empresa era la capacitación del personal, en particular del dedicado a las cabañas y veterinaria.

Finalmente, entre 1930 y el fin de la empresa, el patrimonio se estabiliza en alrededor de 30 millones de pesos. Poseía más de 300.000 hectáreas, y las utilidades rondaron entre el 5\% y el 6\% anual. Esta fue la etapa de madurez de la empresa, donde ya no hay nuevas inversiones. Comparando con la rentabilidad de las empresas industriales, la rentabilidad de Pereda fue mucho menor. La etapa de las grandes utilidades y crecimiento patrimonial había concluido.

\section{Referencias bibliográficas}

ÁLVAREZ, Juan. Temas de historia económica argentina. Buenos Aires: El Ateneo, 1928. BALMORI, Diana;VOSS, Stuart; WORTMAN, Miles Las alianzas de familias y la formación del país en América Latina. México: FCE, 1990.

BARBERO, María Inés. De la Compañía General de Fósforos al grupo fabril. Origen y desarrollo de un grupo económico en la Argentina (1889-1965). En: AAVV. Problemas de investigación, ciencia y desarrollo. San Miguel: Universidad Nacional de General Sarmiento, 2000.

37 Por otro lado, hay que considerar que el ganado, por sus propias características, podría llegar a considerarse como semi-líquido. 
BARSKY, Osvaldo; DJENDEREDJIAN, Julio. Historia del capitalismo agrario pampeano. Buenos Aires: Siglo XXI, 2003.

BOLSA de Comercio de Buenos Aires. Boletín de la Bolsa de Comercio de Buenos Aires. Buenos Aires, 1888-1960.

CASSIS,Youssef; BRAUTASET, Camilla.The performance of European business in the twentieth century: a pilot study. Business and Economic History On-Line, v. 1, 2003. Disponible en < http://www.thebhc.org/sites/default/files/Cassisbrautaset_0.pdf>. Accedido el 1\%/6/2018.

CORTÉS CONDE, Roberto. El progreso argentino. Buenos Aires: Sudamericana, 1979.

CORTÉS CONDE, Roberto. Dinero, deuda y crisis. Buenos Aires: Sudamericana, 1989.

CORTÉS CONDE, Roberto. La economía Argentina en el largo plazo (siglos XIX y XX). Buenos Aires: Sudamericana, 2007.

CUESTA, Eduardo Martín. Celedonio Pereda: patrones de inversión de un gran empresario de la Argentina próspera. Revista de Instituciones, Ideas y Mercados. Buenos Aires, ESEADE, n. 58, p. 79-100, mayo 2013.

CUESTA. Eduardo Martín. Precios, salarios y empresa en la Argentina próspera. El caso del Mercado Central de Frutos (1887-1930).H-industri@, año 8, n. 14, p.121-152, primer semestre 2014.

DE NEVARES, Rodolfo. Jean Estrugamou, un bearnés en Buenos Aires. Buenos Aires: Dunken, 2012.

DJENDEREDJIAN, Julio; SCHMIT, Roberto. Avances y límites de la expansión agraria argentina: crecimiento económico y distribución de la riqueza rural en Entre Ríos, 1860-1890. Investigaciones en Historia Económica. Madrid, n. 11, p. 75-10, 2008.

DURÁN LÓPEZ, Fernando. La autobiografia como fuente histórica: problemas teóricos y metodológicos. Memoria y Civilización. Universidad de Navarra, v. 5, 2002.

GARAVAGLIA, Juan Carlos. Patrones de inversión y elite económica dominante: los empresarios rurales en la pampa bonaerense a mediados del siglo XIX. En: GELMAN, Jorge; GARAVAGLIA, Juan Carlos; ZEBERIO, Blanca (comp.). Expansión capitalista y transformaciones regionales. Buenos Aires: Editorial La Colmena/IEHS, 1999.

GELMAN, Jorge; BARSKY, Osvaldo. Historia del agro argentino. Desde la conquista hasta fines del siglo XX. Buenos Aires: Grijalbo-Mondadori, 2001.

GERCHUNOFF, Pablo; ROCCHI, Fernando; ROSSI, Gastón. Desorden y progreso. Buenos Aires: EDHASA, 2008.

GILBERT, Jorge. Empresario y empresa en la Argentina moderna. El Grupo Tornquist, 1873-1930.Victoria: Universidad de San Andrés, 2002 (Documento de Trabajo, n. 27).

GUSDORF, Georges. Condiciones y límites de la autobiografia. Suplementos Anthropos. Barcelona, n. 29, 1991.

GUTIÉRREZ, Leandro; KOROL, Juan Carlos. Historia de empresas y crecimiento industrial en la Argentina. El caso de la Fábrica Argentina de Alpargatas. Desarrollo Económico, v. 28, n. 111, p. 401-424, oct.-dic. 1988.

GUY, Donna. Refinería Argentina, 1888-1930: límites de la tecnología azucarera en 
una economía periférica. Desarrollo Económico, v. 28, n. 111, p. 353-373, oct.-dic. 1988.

HALPERIN DONGUI, Tulio. La formación de la clase terrateniente bonaerense. Buenos Aires: Prometeo, 2007.

HANSON, Simon. Argentine meat $\mathcal{E}$ the British market. Chapters in the history of the Argentine meat industry. Stanford: Stanford University Press, 1937.

HORA, Roy. La elite social argentina del siglo XIX: algunas reflexiones a partir de la historia de la familia Senillosa. Anuario IEHS. Tandil, UNCPBA, 2002.

HORA, Roy. The making and evolution of the Argentine economic elite: the example of the Senillosas. Hispanic American Historical Review, v. 83, n. 3, p. 451-486, 2003.

HORA, Roy. Los terratenientes de la pampa argentina. Una historia social y política, 18601945. Buenos Aires: Siglo XXI, 2005.

HORA, Roy. Los Anchorena: patrones de inversión, fortuna y negocios (1760-1950). América Latina en la Historia Económica. México, Instituto Mora, v. 37, 2012.

LANCIOTTI, Norma Silvana. Inversión británica y redes empresariales: la estructura organizativa y las estrategias de gestión del Grupo River Plate Trust, Loan \& Agency en Argentina, 1881-1962. Anuario del Centro de Estudios Económicos de la Empresa y el Desarrollo. Buenos Aires, n. 3, p. 85-126, 2011.

LANCIOTTI, Norma Silvana; BARTOLOMÉ, Isabel. Global strategies, differing experiences. Electricity companies in two late-industrialising countries: Spain and Argentina, 1890-1950. Business History, v. 56, n. 5, p. 724-745, 2014.

LLUCH, Andrea. “...Tengo que hacer las veces de médico, comisario, comerciante, defensor de oficio..." Repensando a los comercios rurales de la pampa argentina, 1900-1930. Anuario del Centro de Estudios Históricos, "Prof. Carlos S. A. Segreti". Córdoba, 2003.

LLUCH, Andrea. "De las tierras de América, de la amada Argentina..." Comerciantes minoristas españoles en la pampa argentina (1885-1930). Estudios Migratorios Latinoamericanos. Buenos Aires, 2009.

LOSADA, Leandro. Historia de las elites en la Argentina. Desde la conquista hasta el surgimiento del peronismo. Buenos Aires: Sudamericana, 2009.

MÍGUEZ, Eduardo. Las tierras de los ingleses en la Argentina. Buenos Aires: Sudamericana, 1985.

MÍGUEZ, Eduardo. La expansión agraria de la pampa húmeda (1850-1914).Tendencias recientes de su análisis. Anuario IEHS. Tandil, IHS, n. 1, p. 89-119, 1986.

NEWLAND, Carlos. El sector agropecuario argentino en el período entre siglos (crecimiento económico e intensidad de factores). Una revisión historiográfica. Revista de Historia Económica. Madrid, año XVII, número especial, 1999.

NEWLAND, Carlos. Mercado Mammoth: infraestructura y comercio de productos agropecuarios a través de una empresa argentina, 1887-1916. Revista de Instituciones, Ideas $y$ Mercados. ESEADE, n. 56, 2012.

NEWLAND, Carlos. Eduardo Casey, soñador de empresas. Revista de Instituciones, Ideas y Mercados. ESEADE, n. 58, 2013. 
NEWLAND, Carlos. Sobre las estrategias, utilidades y valuación del Grupo Garovaglio y Zorraquín durante el siglo XX. Revista de Historia Industrial. Barcelona, Universidad de Barcelona, n. 60, p. 81-102, 2015.

NEWLAND, Carlos. Una aproximación microeconómica al cambio estructural argentino, 1926-1945. Desarrollo Económico. Buenos Aires, IDES, vol. 57, n. 222, p. 329-352, 2017.

NEWLAND, Carlos; CUESTA, Martín. Rentabilidad, evolución patrimonial y diversificación en tres grandes compañías argentinas, 1926-1955. América Latina en la Historia Económica. México, Instituto Mora, año 23, n. 2, p. 204-228, 2016.

PALACIO, Juan Manuel. Jorge Sabato y la historiografia rural pampeana: el problema del otro. Entrepasados, Revista de Historia, n. 10, 1996.

PAMPIN, Graciela. La historiografia en torno de la clase dominante. Las tesis de Peña, Sabato y los debates recientes. H-industri@. Buenos Aires, Facultad de Ciencias Económicas, UBA, año 6, n. 10, 2012.

PEREDA, Celedonio. Memorias de la familia Pereda, 1928, manuscrito inédito.

PINEDA, Yovanna. Manufacturing profits and strategies in Argentine industrial development, 1904-1930. Business History, v. 49, n. 2, p. 186-210, 2007.

PUCCIARELLI, Alfredo. El capitalismo agrario pampeano, 1880-1930. Buenos Aires: Hyspamérica, 1986.

RAS, Norberto; LEWIS, Roberto. El precio de la tierra. Buenos Aires: Sociedad Rural Argentina, 1980.

REGALSKY, Andrés. La evolución de la banca privada nacional en Argentina (18801914). Una introducción a su estudio. En: TEDDE, Pedro; MARICHAL, Carlos (coord.). La formación de los bancos centrales en España y América Latina (siglos XIX y XX).V. II. Madrid: Banco Central de España, 1994 (Estudios de Historia Económica, n. 30).

REGUERA, Andrea. Familia, formación de patrimonios y transmisión de la tierra en Tandil (1840-1930). En: GARAVAGLIA, Juan Carlos; GELMAN, Jorge; ZEBERIO, Blanca (comp.). Expansión capitalista y transformaciones regionales. Relaciones sociales y empresas agrarias en la Argentina del siglo XIX. Buenos Aires: IEHS/La Colmena, 1999.

REGUERA, Andrea. Patrón de estancias, Ramón Santamarina: una biografia de fortuna y poder en La Pampa. Buenos Aires: EUDEBA, 2006.

ROCCHI, Fernando. En busca del empresario perdido. Los industriales y las tesis de Jorge Sabato. Entrepasados, Revista de Historia, n. 10, 1996.

ROUGIER, Marcelo. El mercado de valores durante el peronismo. Las dificultades para conformar un ámbito de capitalización empresaria (1944-1955). Investigaciones y Ensayos. Buenos Aires, Academia Nacional de la Historia, n. 59, p. 511-564, 2012.

RUSSO, Cintia. Proceso de trabajo y espacio fabril: el caso de la Cervecería y Maltería Quilmes. Anuario CEEED. Buenos Aires, Facultad de Ciencias Económicas, Universidad de Buenos Aires, año 1, n. 1, p. 203-232, 2009.

SABATO, Hilda. Capitalismo y ganadería en Buenos Aires: la fiebre del lanar, 1850-1890. Buenos Aires: Sudamericana, 1989. 
SABATO, Jorge. La clase dominante en la Argentina moderna. Buenos Aires: CISEA/Grupo Editor Latinoamericano, 1988.

SÁENZ QUESADA, María. Los estancieros. Buenos Aires: Universidad de Belgrano, 1980.

SARTELLI, Eduardo. El enigma de Proteo. A propósito de Jorge F. Sabato, Larry Sawers y el estancamiento de la economía argentina. Ciclos, año VI, v.VI, n. 10, 1996.

SCHMIT, Roberto. Estancias de Entre Ríos en el siglo XIX, 1840 y 1880. Mundo Agrario, v. 16, n. 31, 2015. Disponible en <http://www.mundoagrario.unlp.edu.ar/ article/view/MAv16n31a01>. Accedido el 1/6/2018.

SCHMIT, Roberto; DJENDEREDJIAN, Julio. La empresa rural en el largo plazo. Cambios en la explotación de una gran estancia rioplatense entre el orden colonial y el nacimiento del capitalismo, 1780-1870. Boletín del Instituto de Historia Argentina y Americana Dr. E. Ravignani. Buenos Aires, FFyL, EUDEBA, n. 29, p. 7-49, 2007.

SCHVARZER, Jorge. Terratenientes, industriales y clase dominante en el ya antiguo debate sobre el desarrollo argentino. Desarrollo Económico, v. 41, n. 161, p. 121-126, 2001.

SESTO, Carmen. La vanguardia ganadera bonaerense, 1856-1900: historia del capitalismo agrario pampeano. Buenos Aires: Siglo XXI/Universidad de Belgrano, 2005.

SOCOLOW, Susan. Los mercaderes del Buenos Aires virreinal. Buenos Aires: De la Flor, 1991.

TAFUNELL, Xavier. La rentabilidad financiera de la empresa española, 1880-1981: una estimación en perspectiva sectorial. Revista de Historia Industrial, n. 18, p. 71-112, 2000 . 